\title{
Dissipation-driven quantum phase transition in superconductor-graphene systems
}

\author{
Roman M. Lutchyn, ${ }^{1}$ Victor Galitski, ${ }^{1}$ Gil Refael, ${ }^{2}$ and S. Das Sarma ${ }^{1}$ \\ ${ }^{1}$ Joint Quantum Institute and Condensed Matter Theory Center, \\ Department of Physics, University of Maryland, College Park, MD 20742-4111, USA \\ ${ }^{2}$ Department of Physics, California Institute of Technology, MC 114-36, Pasadena, CA 91125
}

(Dated: March 9, 2022)

\begin{abstract}
We show that a system of Josephson junctions coupled via low-resistance tunneling contacts to graphene substrate(s) may effectively operate as a current switching device. The effect is based on the dissipation-driven superconductor-to-insulator quantum phase transition, which happens due to the interplay of the Josephson effect and Coulomb blockade. Coupling to a graphene substrate with gapless excitations further enhances charge fluctuations favoring superconductivity. The effect is shown to scale exponentially with the Fermi energy in graphene, which can be controlled by the gate voltage. We develop a theory, which quantitatively describes the quantum phase transition in a two-dimensional Josephson junction array, but it is expected to provide a reliable qualitative description for one-dimensional systems as well. We argue that the local effect of dissipationinduced enhancement of superconductivity is very robust and a similar sharp crossover should be present in finite Josephson junction chains.
\end{abstract}

Artificially fabricated Josephson-junction arrays (JJA) offer a unique playground for studying quantum phase transitions (QPT) [1]. The transitions in JJA occur due to the competition between the Josephson coupling, $E_{J}$, which favors a globally ordered state, and the charging energy, $E_{c}$, which leads to Coulomb blockade of Cooper-pair tunneling and enhances quantum fluctuations of the superconducting (SC) phase. At zero temperature, the QPT from a globally superconducting to an insulating phase [2, 3, 4, 5, 6] occurs, roughly, when the Josephson energy $E_{J}$ becomes smaller than the charging energy. Another key factor in determining the ground state is dissipation, which is present whenever the SC system is connected to a reservoir of gapless single-particle excitations [7]. The main effect of the dissipation in JJA is a suppression of quantum phase fluctuations. Taking into account the phasecharge uncertainty relation, the dissipation enhances fluctuations of the charge and, hence, stabilizes the SC phase[8]. This type of dissipative QPT has been considered previously by Feigelman and Larkin [9] in the model of a regular 2D proximity-coupled JJA and by Galitski and Larkin [10] in a disorder-induced random Josephson network. In both cases, it was found that the effect of dissipation on the transition point is exponential, i.e., the critical Josephson coupling scales exponentially with the Andreev conductance. The Andreev conductance and hence the degree to which the stabilizing effect of dissipation is important obviously depend on the density of states of gapless excitations. Thus, by controlling the latter, one can tune transitions between a global superconductor and an insulator. This observation provides strong motivation for studying superconductors coupled to a graphene substrate, where the density of carriers can be easily tuned by gate voltage from essentially zero at the Dirac point (no "Ohmic" dissipation) to very large values in the electron-doped graphene (strong dissipation).

In this Letter, we propose to study the superconductorinsulator phase transition (SIT) in a Josephson-junction array in a tunneling contact with a graphene layer (or layers) (see Fig. 11, which acts as a source of gapless quasiparticles.
The graphene substrate provides a unique possibility to control the dissipation strength via the gate voltage and thereby tune the dissipation-driven QPT. Hence, the system may be used as a current switching device. While the physics of the underlying effect is intuitively quite clear, the formal description of the transition developed in this Letter is technically non-trivial: First, we use the tunneling Hamiltonian formalism and elements of random matrix theory to derive the effective phase fluctuation action of a small SC grain coupled to a graphene substrate. The dissipation kernel, $K(\tau)$, shows a crossover from the Ohmic dissipation behavior $K(\tau) \propto \tau^{-2}$ in the electron-doped graphene to extremely weak dissipation $K(\tau) \propto \tau^{-4}$ at the Dirac point. Second, we develop a meanfield theory of the SIT and show that the quantum critical point is determined by the single-grain phase correlator, which is to be calculated using the dissipative effective action. To calculate the phase correlator we use the two-loop renormalization group (RG) results from a related spin model and determine a critical voltage, $V_{c}$, at which the transition occurs: For $V>V_{c}$, the system is a superconductor; for $V<V_{c}$, the system is an insulator.

Our theoretical model is an array of SC grains connected with each other with the Josephson junctions and connected via tunnel contacts to a graphene substrate, see Fig. 1 For $T \rightarrow 0$, one can neglect massive fluctuations of the amplitude of the order parameter $\Delta$ in the grain, and describe the dynamics of the system in terms of the phase-only imaginary-time effective action $(\hbar=1)$

$$
S_{C}+S_{J}=\sum_{i} \int d t \frac{\dot{\varphi}_{i}(\tau)^{2}}{E_{c}}-\sum_{<i j>} \int d t E_{J} \cos \left[\varphi_{i}(\tau)-\varphi_{j}(\tau)\right],
$$

where $\varphi_{i}$ is the phase of the order parameter on the $i$-th grain. Here, for simplicity, we assume that the Josephson and charging energy are the same for all grains. However, this assumption is not essential for our results.

We now consider the effect of graphene gapless excitations on the phase coherence of a single SC grain. We study here the situation when the $\mathrm{SC}$ grain of radius $R$ lies on top of the 


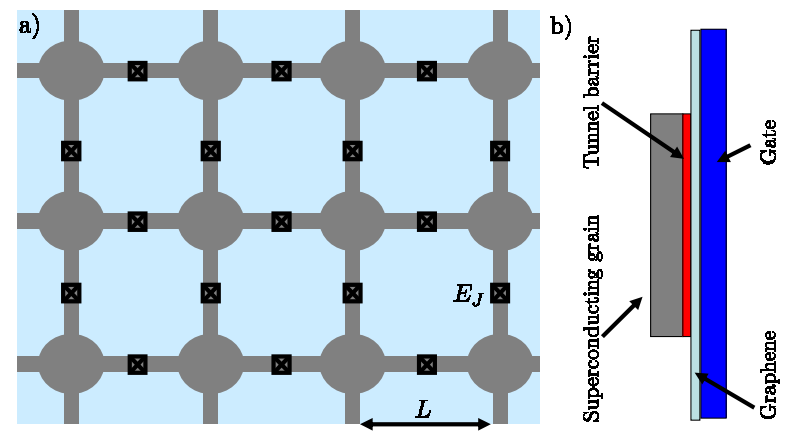

FIG. 1: (Color online) a) A Josephson-junction array on top of a graphene sheet is shown. The grains are coupled by the Josephson junctions with the coupling strength $E_{J}$. The distance between the grains $L$ is assumed to be large compared to the SC coherence length $\xi$. Thus, the coherent transport through the graphene is neglected here. Alternatively, the SC grains in the array can be coupled to different graphene sheets. Then, there is no coherent transport through the graphene due to physical separation between the sheets. b) The superconductor/graphene interface. The graphene and superconductor are connected through the tunnel barrier. The chemical potential in graphene can be tuned with the gate voltage $V_{g}$.

graphene sheet, see Fig. 1. In this planar geometry the tunnel junction does not break internal symmetries of graphene, and thus does not modify the spectrum of the excitations. In the limit of low transparency tunnel barrier, the transport between superconductor and graphene can be described by the tunneling Hamiltonian

$$
H_{T}=t \sqrt{d_{z}} \sum_{\sigma} \int_{A} d^{2} \boldsymbol{r}\left[\Psi_{\sigma}^{\dagger}(\boldsymbol{r}) \Psi_{\sigma}^{(g)}(\boldsymbol{r})+\text { H.c. }\right],
$$

where $\Psi_{\sigma}^{(g)}(\boldsymbol{r})$ and $\Psi_{\sigma}(\boldsymbol{r})$ are the electron operators in graphene and superconductor, respectively. Here $d_{z}$ is the thickness of the grain, and $t$ and $A$ are the tunneling matrix element and the area of the junction, respectively.

Assuming that the SC gap energy is sufficiently large, the main contribution to the subgap transport originates from Andreev processes, which involve correlated tunneling of two electrons from/to the graphene. In the fourth order perturbation theory in tunneling $t$ (see Fig. 22), the contribution of the Andreev processes to the dynamics of the phase is given by the following effective action

$$
\begin{aligned}
S_{A} & =24 t^{4} d_{z}^{2} \int_{A} \prod_{i=1 . .4} d \boldsymbol{x}_{i} \\
& \operatorname{Re}\left[F^{*}\left(\boldsymbol{x}_{1}, \boldsymbol{x}_{2}\right) F\left(\boldsymbol{x}_{3}, \boldsymbol{x}_{4}\right) G^{(g)}\left(\boldsymbol{x}_{1}, \boldsymbol{x}_{3}\right) G^{(g)}\left(\boldsymbol{x}_{2}, \boldsymbol{x}_{4}\right)\right],
\end{aligned}
$$

with $\boldsymbol{x}=\{\boldsymbol{r}, \tau\}$. Here $G^{(g)}\left(\boldsymbol{x}, \boldsymbol{x}^{\prime}\right)$ and $F\left(\boldsymbol{x} ; \boldsymbol{x}^{\prime}\right)$ are imaginarytime Green's functions for graphene and superconductor, respectively. The latter is defined as

$$
\begin{gathered}
F^{*}\left(\boldsymbol{r}_{1}, \boldsymbol{r}_{2} ; \tau_{1}, \tau_{2}\right)=-e^{i\left[\phi\left(\tau_{1}\right)+\phi\left(\tau_{2}\right)\right] / 2} \sum_{n} \chi_{n}\left(\boldsymbol{r}_{1}\right) \chi_{n}\left(\boldsymbol{r}_{2}\right) u_{n} v_{n} \\
{\left[\Theta\left(\tau_{1}-\tau_{2}\right) e^{-\left(\tau_{1}-\tau_{2}\right) E_{n}} \Theta\left(E_{n}\right)-\Theta\left(\tau_{2}-\tau_{1}\right) e^{-\left(\tau_{1}-\tau_{2}\right) E_{n}} \Theta\left(-E_{n}\right)\right] .}
\end{gathered}
$$

Here $E_{n}=\sqrt{\varepsilon_{n}^{2}+\Delta^{2}}, u_{n}$ and $v_{n}$ are Bogoliubov coherence factors $u_{n}^{2} / v_{n}^{2}=\left(1 \pm \varepsilon_{n} / E_{n}\right) / 2$, and $\varepsilon_{n}$ and $\chi_{n}(\boldsymbol{r})$ are the eigenvalues and eigenfunctions of the single particle Hamiltonian of the grain, which includes random potential due to impurities and boundaries of the island.

In order to derive low-energy effective action $S_{A}$ due to Andreev processes, it is convenient to separate the fast and slow times $\kappa$ and $\tau: \tau_{1}=\tau+\kappa / 2$ and $\tau_{2}=\tau-\kappa / 2$. Since the superconducting Green's function decays exponentially on the time scale of order $\Delta^{-1}$, one can neglect $\kappa$ in the dynamics of the phase, which evolves on much longer time scales. Taking advantage of this separation of scales and performing the integral over the fast time scales, we obtain the following expression for $S_{A}$

$$
\begin{aligned}
S_{A} & \approx 24 t^{4} d_{z}^{2} \int_{A_{i=1 . .4}} d \boldsymbol{r}_{i} d \tau d \tau^{\prime} \cos \left[\phi(\tau)-\phi\left(\tau^{\prime}\right)\right] \\
& \times F_{12} F_{34} G_{13}^{(g)}\left(\tau-\tau^{\prime}\right) G_{24}^{(g)}\left(\tau^{\prime}-\tau\right) .
\end{aligned}
$$

Here $F_{i j}$ is the anomalous Green's function at zero frequency, i.e $F_{i j}=-\sum_{n} \chi_{n}\left(\boldsymbol{r}_{i}\right) \chi_{n}\left(\boldsymbol{r}_{j}\right) \Delta / E_{n}^{2}$.

It is well-known that Andreev transport is sensitive to disorder [11]. Therefore, in order to calculate the effective action, one has to take into account spatial correlations [11, 12, 13], and average the action over the random realization of the wavefunctions in the SC grain resulting from the scattering of electrons from the grain boundaries and impurities. We perform this averaging using exact eigenstates technique assuming that the grain is sufficiently small. Our approach accounts for the enhancement of the tunneling rate due to the coherent back-scattering of electrons to the tunnel junction. The correlation function $\left\langle F_{12} F_{34}\right\rangle$ in the grain consists of reducible and irreducible parts:

$$
\left\langle F_{12} F_{34}\right\rangle \approx\left\langle F_{12}\right\rangle\left\langle F_{34}\right\rangle+\left\langle F_{12} F_{34}\right\rangle_{i r} .
$$

The reducible part can be easily calculated

$$
\left\langle F_{12}\right\rangle=\frac{\pi}{2} v_{F} f_{12} \text { with } f_{12}=\frac{\sin \left(k_{F}\left|\boldsymbol{r}_{1}-\boldsymbol{r}_{2}\right|\right)}{k_{F}\left|\boldsymbol{r}_{1}-\boldsymbol{r}_{2}\right|} e^{-\frac{\left|\boldsymbol{r}_{1}-\boldsymbol{r}_{2}\right|}{2 l}},
$$

where $k_{F}, l$ and $v_{F}$ are the Fermi wave vector, mean free path, and density of states at the Fermi level in the grain, respectively.

The irreducible correlation function is calculated assuming that $\mathrm{SC}$ grains are small, and the Thouless energy $E_{T}$ is the largest relevant energy scale in the problem $E_{T} \sim D / R^{2} \gg$ $\Delta, E_{c}, E_{J}$, where $D$ is the diffusion constant, $R$ is the radius of the grain, and $\delta$ is the level spacing in the grain. In this limit, the electron diffusion time in the grain $\tau_{D}=1 / E_{T}$ is much smaller than the time the system dwells in the virtual state with one unpaired electron $\sim 1 / \Delta$. Since an electron in the virtual state covers the entire available phase space, one can calculate the irreducible correlation function within Random Matrix Theory [14] finding that it acquires the universal form $\left\langle F_{12} F_{34}\right\rangle_{i r}=\frac{\pi}{4} \frac{v_{F}^{2} \delta}{\Delta}\left(f_{14} f_{23}+f_{13} f_{24}\right)$. Combining this equation 


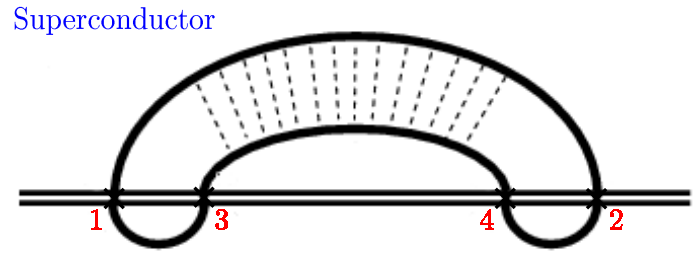

Graphene

FIG. 2: The diagrams describing the correlated two-electron tunneling process (Andreev process) from/to the SC grain are shown.

with Eq. (6), we obtain

$$
\left\langle F_{12} F_{34}\right\rangle=\frac{\pi^{2}}{4} v_{F}^{2} f_{12} f_{34}+\frac{\pi}{4} \frac{\delta}{\Delta} v_{F}^{2}\left(f_{14} f_{23}+f_{13} f_{24}\right) .
$$

Using Eqs. (7) and (4), and carrying out the spatial integrals, we find

$$
S_{A} \approx \frac{6 \pi t^{4}}{\delta \Delta k_{F}^{4}} \int d \tau d \tau^{\prime} \cos \left[\phi(\tau)-\phi\left(\tau^{\prime}\right)\right] G_{11}^{(g)}\left(\tau-\tau^{\prime}\right) G_{22}^{(g)}\left(\tau^{\prime}-\tau\right) .
$$

This term originates from the integration of the irreducible correlation function and is parametrically larger than the reducible term by the factor of $k_{F}^{2} A \delta / \Delta \gg 1[11,13]$. After substituting the expressions for the graphene Green's functions (see, e.g., Ref. [15]) and assuming that $\mu \geq 0$, we find

$S_{A} \approx-G \int d \tau d \tau^{\prime} K\left(\tau-\tau^{\prime}\right) \cos \left[\phi(\tau)-\phi\left(\tau^{\prime}\right)\right], G=\frac{3 t^{4}}{2 \pi \delta \Delta k_{F}^{2} \gamma^{2}}$,

where $\gamma$ is the Fermi-velocity in graphene. The kernel $K(\tau)$ is described by the following function

$$
K(\tau)=\frac{1}{k_{F}^{2} \gamma^{2} \tau^{4}}\left\{(\mu \tau)^{2}+2[1-\mu|\tau|] e^{-\mu|\tau|}-1\right\},
$$

which exhibits a crossover from $\tau^{-4}$ at the Dirac point to the usual Ohmic behavior $\tau^{-2}$ at $\mu|\tau| \sim 1$. For realistic experimental parameters the chemical potential $\mu$ is much larger than the characteristic timescale for the dynamics of the phase $\tau \leq E_{c}^{-1}$, i.e. $\mu \gg E_{c}$. Thus, the asymptotic form of the effective action becomes

$$
S_{A} \approx-\eta \int d \tau d \tau^{\prime} \frac{\cos \left[\phi(\tau)-\phi\left(\tau^{\prime}\right)\right]}{\left(\tau-\tau^{\prime}\right)^{2}}, \quad \eta=G \frac{\mu^{2}}{k_{F}^{2} \gamma^{2}}
$$

The important difference between action (11) and the dissipative action describing resistively-shunted Josephson junction, is that action (11) is "compact" or periodic in the phase difference, and thus correctly describes the fact that the charge on the grain is quantized and can change by $2 e$ only. From Eq. (11), we see that graphene as a source of gapless excitations provides the possibility to change the dissipation strength directly by changing the chemical potential, which is tied to the gate voltage.

Dissipation-driven QPT. Combining Eqs. (1) and (11), we obtain the full action for the system: $S=S_{C}+S_{J}+S_{A}$. To derive effective action describing the transition in the JJA, we first write a partition function in path integral representation and then use Hubbard-Stratonovich transformation to decouple Josephson term by introducing an auxiliary field $\psi_{i}(\tau)$ coupled linearly to $e^{i \varphi_{i}(\tau)}$. Then, the partition function becomes $Z=Z_{0} \int \mathfrak{D} \psi \exp (-S[\psi])$, where $S[\psi]$ is given by

$$
\begin{aligned}
S[\psi]= & \int d \tau \frac{1}{2} \sum_{i, j} \psi_{i}^{*}(\tau) w_{i j}^{-1} \psi_{j}(\tau) \\
& -\ln \left\langle\exp \left[\frac{1}{2} \int d \tau \sum_{i} e^{i \varphi_{i}(\tau)} \psi_{i}^{*}(\tau)+\text { c.c. }\right]\right\rangle_{0} .
\end{aligned}
$$

Here we introduced the symmetric matrix $w_{i j}$, which describes Josephson tunneling: matrix elements of $w_{i j}$ are equal to $E_{J}$ for nearest neighbors and zero otherwise. The expectation value in Eq. (12) is taken with respect to the single-site action $S_{0}=S_{C}+S_{A}$. To study QPT at the mean field level, one may perform cumulant expansion of the second term in the action $S[\psi]$ in powers of $\psi$ and arrive at the effective complex $\psi^{4}$ field theory [16]. The phase boundary between macroscopically superconducting and insulating state of JJA can be obtained by setting the coefficient $r$ in front of $|\psi|^{2}$ to zero:

$$
r \propto \frac{1}{z E_{J}}-\frac{1}{2} \int d \tau\left\langle e^{i \varphi(\tau)-i \varphi(0)}\right\rangle_{0}=0 .
$$

Here $z$ is the coordination number of the lattice and averaging is taken with respect to $S_{0}$. When calculating the correlation function we assume that the dissipation strength $\eta$ is large. Then, the second term in $S_{0}$ dominates at low frequencies and one can neglect the influence of the charging energy term $S_{C}$, which serves as the ultra-violet cut-off. Under these conditions, the correlation function can be mapped on the long-range ferromagnetic spin chain [17, 18, 19] first considered by Kosterlitz [17]. Later, the critical behavior was studied in Ref. [19, 20], where the asymptotic behavior of the spin-spin correlation function was obtained using two-loop renormalization group (RG). Adopting the results of Refs. [9, 17, 19, 20] to our problem, we get

$$
\left\langle e^{i \varphi(\tau)-i \varphi(0)}\right\rangle_{0} \sim \begin{cases}\left(\frac{\tau_{c}}{\tau}\right)^{\frac{1}{2 \pi^{2} \eta}}, & \Lambda^{-1} \ll \tau \ll \tau_{c} \\ \left(\frac{\tau_{c}}{\tau}\right)^{2}, & \tau \gg \tau_{c},\end{cases}
$$

where $\Lambda$ is the ultraviolet cut-off, $\Lambda \sim 2 \pi E_{c} \eta$, and $\tau_{c}$ is the correlation time, which can be calculated using the RG for $\eta$. Since the compact dissipation term proportional to $\eta$ is not Gaussian, it gets renormalized when integrating out highfrequency degrees of freedom resulting in the following flow equations

$$
\frac{d \eta}{d \ln (\Lambda \tau)}=-\frac{1}{2 \pi^{2}}-\frac{1}{\left(2 \pi^{2}\right)^{2} \eta},
$$

where the rhs is the beginning of a Taylor expansion in $1 / \eta$. By integrating this equation between the initial value $\eta(0) \equiv \eta$ 


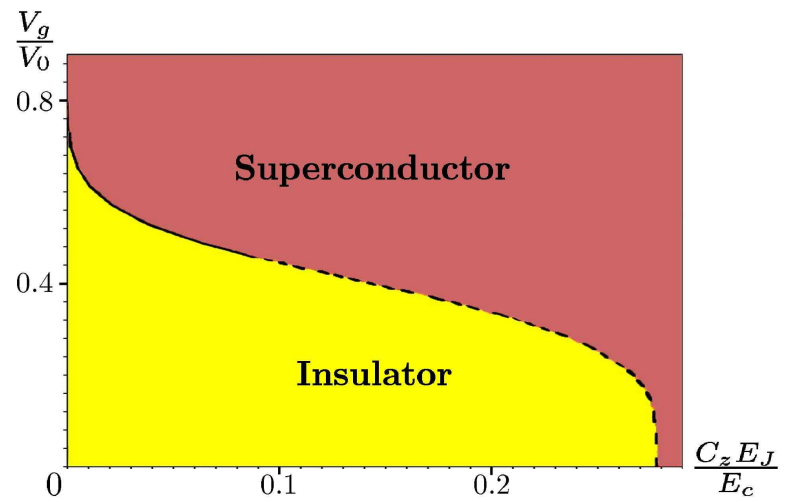

FIG. 3: (Color online). Phase diagram for the SIT is shown. By tuning the applied gate voltage $V_{g}$, one can change the density of states of gapless excitations in graphene, and thus drive the phase transition between globally superconducting and insulating states of JJA. The solid (black) line represents the region of the validity of RG.

and final value $2 \pi^{2} \eta\left(\ln \left[\Lambda \tau_{c}\right]\right) \sim 1$, we can estimate the correlation time as

$$
\tau_{c} \sim\left(4 \pi^{3} E_{c} \eta^{2}\right)^{-1} \exp \left(2 \pi^{2} \eta\right) .
$$

It is assumed here that $2 \pi^{2} \eta \gg 1$. Using Eqs. (14) and (16) and carrying out the time integral, we find expression defining the phase boundary $C_{z} E_{J}=4 \pi^{3} E_{c} \eta_{c}^{2} \exp \left(-2 \pi^{2} \eta_{c}\right)$, where $C_{z}$ is a numerical constant depending on the coordination number $z$. The rhs of the above equation corresponds to the effective charging energy of the grain $E_{c}^{*}$ renormalized by the dissipation [21, 22, 23]. The critical dissipation strength, $\eta_{c}$, and the corresponding critical voltage, $V_{c}$, at which the QPT occurs can be expressed via Lambert-W function by inverting the transcendent algebraic equation above:

$$
\frac{V_{c}}{V_{0}}=\frac{1}{\pi} \sqrt{-W_{-1}\left(-\sqrt{\frac{\pi C_{z} E_{J}}{4 E_{c}}}\right) .}
$$

Here $V_{0}=\frac{\gamma k_{F}}{e \sqrt{G}}$ with $k_{F}, \gamma$ and $G$ being the Fermi-momentum in the SC grains, the Fermi-velocity in graphene, and the dimensionless constant defining the transparency of the tunnel barrier (see Eq. (9)), respectively. The phase boundary between globally superconducting and insulating phases of JJA is shown in Fig. 3. For the theory based on the RG procedure to be formally valid, we need $\ln \left(E_{c} / E_{J}\right) \gg 1$. This, however, is a mathematical rather than physical constraint and for all practical purposes (i.e., experiment) what is important is the existence of the transition itself; the applicability of RG methods and the exact location of the non-universal "critical voltage" are not essential. A sharp transition should certainly exist if $E_{c} \gg E_{J}$ and perhaps even for $E_{c} \geq E_{J}$. In the opposite limit the system is already superconducting without any substrate and there is no dissipation-driven effect. Since the technology for making SC grains with required $E_{c} / E_{J}$ is welldeveloped, the conditions for the observation of the QPT are certainly experimentally feasible.
We also emphasize here that since the dissipation-driven transition is intrinsically local, it is expected to survive in one-dimensional chains (where the mean-field theory breaks down and the QPT is of Kosterlitz-Thouless type, see, e.g., Ref. [24]). Again, the exact location of the transition point and the critical behavior would be different, but the effect itself should be present. Moreover, the same local argument suggests that a sharp voltage-induced crossover (cf. with the model of a shunt resistor coupled locally to the SC grain [25, 26]) in the IV-curves should be present even in finite chains proximity coupled to graphene, similar to those that are already being experimentally investigated [27, 28]. We propose that experiments be carried out in the SC-graphene system to directly confirm our quantum phase transition and current switching predictions.

Acknowledgments. We thank M. Feigel'man, E. Hwang, J. Lau and S. Tewari for stimulating discussions. VG acknowledges the hospitality of Boston University visitors program. This work has been supported (RML and SDS) by US-ONR and LPS-NSA-CMTC.

[1] A. M. Goldman and N. Markovic, Physics Today 51, 39 (1998).

[2] I. S. Beloborodov et al., Rev. Mod. Phys. 79, 469 (2007).

[3] B.G. Orr et al., Phys. Rev. Lett. 56, 378 (1986).

[4] H. S. J. van der Zant et al., Phys. Rev. B 54, 10081 (1996).

[5] Y. Takahide et al., Phys. Rev. Lett. 85, 1974 (2000).

[6] A. J. Rimberg et al., Phys. Rev. Lett. 78, 2632 (1997).

[7] G. Schoen and A. D. Zaikin, Phys. Rep. 198, 237 (1990).

[8] S. Chakravarty et al., Phys. Rev. Lett. 56, 2303 (1986).

[9] M. V. Feigel'man and A. I. Larkin, Chem. Phys. 235, 107 (1998).

[10] V. Galitski and A. I. Larkin, Phys. Rev. Lett. 87, 087001 (2001).

[11] F. W. J. Hekking and Yu. V. Nazarov, Phys. Rev. B 49, 6847 (1994).

[12] C. Bruder, R. Fazio and G. Schoen, Physica B 203240 (1994).

[13] R. M. Lutchyn and L. I. Glazman, Phys. Rev. B 76, 104507 (2007).

[14] I. L. Aleiner and P. W. Brouwer and L. I. Glazman, Physics Reports 358, 309 (2002).

[15] J. Gonzalez and E. Perfetto, Phys. Rev. B 76, 155404 (2007).

[16] S. Sachdev, Quantum Phase Transitions (Cambridge University Press, 1999).

[17] J. M. Kosterlitz, Phys. Rev. Lett. 37, 1577 (1976).

[18] E. Brezin, J. Zinn-Justin and J. C. Le Guillou, J. Phys. A 9, L119 (1976).

[19] W. Hofstetter and W. Zwerger, Eur. Phys. J. B 5, 751 (1998).

[20] H. Spohn and W. Zwerger, J. Stat. Phys. 94, 1037 (1999).

[21] S.V. Panyukov, A.D. Zaikin, Phys. Rev. Lett. 673168 (1991).

[22] G. Falci, G. Schoen, and G.T. Zimanyi, Phys. Rev. Lett. 74, 3257 (1995).

[23] S. L. Lukyanov and P. Werner, J. Stat. Mech. 11, 11002 (2006).

[24] J. S. Meyer, A. Kamenev, and L. I. Glazman, Phys. Rev. B 70, 45310 (2004).

[25] Gil Refael et al., Phys. Rev. B 75, 014522 (2007).

[26] S. Tewari and J. Toner, Europhys. Lett., 74, 341 (2006).

[27] H. B. Heersche et al, Nature 446, 56 (2007).

[28] Xu Du et al., arXiv.org:0710.4984. 